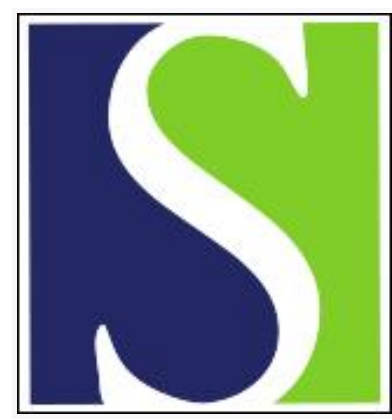

Scand J Work Environ Health 1999;25(3):215-221

https://doi.org/10.5271/sjweh.426

Issue date: Jun 1999

Risk of premenopausal breast cancer in association with occupational exposure to polycyclic aromatic hydrocarbons and benzene

by Petralia SA, Vena JE, Freudenheim JL, Dosemeci M, Michalek A, Goldberg MS, Brasure J, Graham S

The following articles refer to this text: 2018;44(3):310-322;

2021;47(2):154-162

Key terms: benzene; estrogen receptor; occupational exposure; polycyclic aromatic hydrocarbon; premenopausal breast cancer; woman

This article in PubMed: www.ncbi.nlm.nih.gov/pubmed/10450771

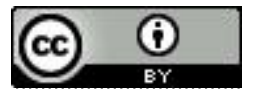




\title{
Risk of premenopausal breast cancer in association with occupational exposure to polycyclic aromatic hydrocarbons and benzene
}

\author{
by Sandra A Petralia, PhD, ${ }^{1,2}$ John E Vena, PhD, ${ }^{1}$ Jo L Freudenheim, PhD, ${ }^{1}$ Mustafa Dosemeci, PhD ${ }^{2}$ \\ Arthur Michalek, PhD, ${ }^{1}$ Mark S Goldberg, PhD, ${ }^{3}$ John Brasure, BS, ${ }^{1}$ Saxon Graham, PhD'
}

\begin{abstract}
Petralia SA, Vena JE, Freudenheim JL, Dosemeci M, Michalek A, Goldberg MS, Brasure J, Graham S. Risk of premenopausal breast cancer in association with occupational exposure to polycyclic aromatic hydrocarbons and benzene. Scand J Work Environ Health 1999;25(3):215-221.
\end{abstract}

\begin{abstract}
Objectives This study examined the relationship between risk of premenopausal breast cancer and occupational exposure to benzene and polycyclic aromatic hydrocarbons $(\mathrm{PAH})$ and whether the proposed relationship between $\mathrm{PAH}$ and breast cancer differed by tumor estrogen receptor (ER) status.

Methods In a case-referent study of premenopausal breast cancer, occupational histories and other information were obtained through interviews, and job-exposure matrices were used to assess exposure to PAH and benzene. Results A dose-response relationship for the probability of exposure to benzene [low: odds ratio (OR) 1.64, 95\% confidence interval $(95 \% \mathrm{CI}) 0.64-4.21$; high: OR $1.95,95 \% \mathrm{CI} 1.14-3.33$ ) and to PAH (low: OR 1.56, 95\% CI 0.78-3.12; high: OR 2.40,95\% CI 0.96-6.01). Risk increased with duration of exposure to benzene, but not to PAH. A dose-response relationship was not evident for the intensity of exposure to benzene or to PAH. When analyses were stratified by tumor ER status, PAH exposure was related to a greater increase in the risk of ERpositive (OR 2.27, 95\% CI 1.14 -4.54) than ER-negative (OR 1.12, 95\% CI 0.47-2.64) breast cancer. Risk of ER-positive, but not ER-negative, tumors increased with the probability of exposure to PAH.

Conclusions The findings suggest an association between risk and occupational exposure to benzene. Although it was difficult to study PAH independently of benzene, there was some suggestion of an association between PAH exposure and ER-positive tumors. These data should be interpreted with caution because of the limitations of this study, including low-response rates and small numbers of exposed persons.
\end{abstract}

Key terms benzene, breast cancer, estrogen receptor, occupational exposures, polycyclic aromatic hydrocarcarbons, women.

Aromatic hydrocarbons such as benzene and the polycyclic aromatic hydrocarbons $(\mathrm{PAH})$, benzo[a]pyrene $(\mathrm{BaP})$ dibenz[ah]anthracene (DB[ah]A) and 7,12-dimethylbenz[a]anthracene (DMBA) are mammary carcinogens in animals (1), and they have been proposed as human breast carcinogens $(1,2)$, the epidemiologic evidence is limited. Lipophilic aromatic hydrocarbons are stored in mammary fat tissue. They are metabolized by the P-450 enzyme system into metabolites that may promote carcinogenesis. Workers exposed to $\mathrm{BaP}$ were found to have high levels of BaP-DNA adducts (3), and breast cancer patients have been found to have higher levels of PAHDNA adducts (DNA $=$ deoxynucleic acid) when compared with cancer-free referents (4). PAH-DNA adducts have also been shown to correlate with chromosome mutations in human populations exposed environmentally to PAH (5). Occupational exposure to benzene has been linked to chromosome aberrations $(6,7)$ and sister chromatid exchanges (7). Furthermore, experimental investigations have demonstrated that $\mathrm{BaP}$ and DMBA possess estrogenic properties (8-10) and may be especially relevant for tumors rich in estrogen receptors (ER). Dewailly et al (11) found that ER-positive, but not ERnegative cases had higher adipose and plasma concentrations of several estrogenic organochlorines than referents. When all the cases were combined, no differenc-

1 Department of Social and Preventive Medicine, School of Medicine and Biomedical Sciences, State University of New York at Buffalo, Buffalo, New York, United States.

2 National Cancer Institute, Bethesda, Maryland, United States.

3 Institut Armand-Frappier, University of Québec, and Joint Departments of Epidemiology and Biostatistics and Occupational Health, McGill University, Montreal, Quebec, Canada.

Reprint requests to: Dr Sandra A Petralia, Occupational Epidemiology Branch, National Cancer Institute, Executive Plaza South Rm 8110, 6120 Executive Blvd, Bethesda, MD 20892, United States. [E-mail: sp126i@ nih.com] 
es were found. In another study, a positive correlation was detected between concentrations of DDE in breast adipose tissue of breast cancer patients and ER concentrations (12).

Breast cancer risk in relation to benzene and $\mathrm{PAH}$ exposure has not been widely investigated in occupational epidemiologic studies, and the results have been conflicting (13-17).

Through the use of occupational histories and job exposure matrices, we evaluated the relationship of occupational exposure to the aromatic hydrocarbons, benzene and PAH with ER status and breast cancer risk in premenopausal women.

\section{Subjects and methods}

\section{Study population and data collection}

The data used for this research derive from a case-referent study conducted in western New York State (18). To be included in the study, women had to be 40 years of age or older with at least one functioning ovary. Incident, primary, histologically confirmed premenopausal breast cancer cases were identified from all major hospitals in the Erie and Niagara counties in New York State between 1986 and 1991. The referents were selected randomly from lists of the New York State Department of Motor Vehicles and were frequency-matched to cases by age and county of residence. Overall, 66\% (301) of the identified eligible cases and $62 \%$ (316) of the identified eligible referents participated in the study. All the participants signed an informed written consent granting us an interview; the cases also gave us permission to review their hospital records.

Personal interviews were conducted which included questions regarding occupational history, reproductive, menstrual and medical histories, and other factors. For each job held for 1 year or longer, the participants provided job title, duties, the calendar years during which the job was held and whether it was full- or part-time, the employer's name, and a description of what the company manufactured or the service it provided. Occupational titles and industries were coded according to codes of the United States (US) 1980 Bureau of Census (19).

ER information was abstracted from pathology reports and medical records for $76.4 \%$ of the cases. Cases with missing ER information did not differ from those with ER status by occupational exposure to PAH or benzene, or by other characteristics aside from having a slightly lower average Quetelet index and being less likely to have smoked cigarettes (data not shown). For the majority of cases whose ER data were available, the ER concentration was determined using the dextran-coated charcoal method (DCC) (93.5\%). If the tissue samples were smaller than the 0.5 grams required for this assay, they were analyzed using the immunoperoxidase cytochemical assay ( $\mathrm{N}=6$, or $2.6 \%)$ or the fluorocytochemical assay $(2.9 \%)$. The assays were performed at the Buffalo General Hospital laboratory (76.1\%), Roche laboratories $(16.5 \%)$ or Nichols laboratories (4.3\%), and, for $3 \%$ of the cases with ER status, information on the assay and laboratory used was missing. Quantitative measures (measured as femtomoles per milligram of cytosol protein when the DCC method was used or as the percentage of tumor cells positive for estrogen receptors when cytochemical methods were used) and qualitative information (positive or negative) on estrogen receptors was reported. Tumors with an ER concentration value greater than $10 \mathrm{fmol} / \mathrm{mg}$ or with greater than $10 \%$ of the cells with estrogen receptors were classified as ER-positive.

\section{Exposure assessment}

Job-exposure matrices for PAH and benzene, developed by one of the authors (MD), were used to determine which occupations and occupation and industry combinations involved potential exposure. The methods used to develop these exposure estimates were similar to those used for previously developed matrices for exposure to chlorinated aliphatic hydrocarbons (20). For each matrix, each 3-digit 1980 code of the US Bureau of Census occupation and industry (19) was evaluated for potential exposure to the agent of interest on the basis of data from the literature, unpublished industrial hygiene reports and inspections, and the industrial hygienist's expert opinion.

Each occupation and industry code was assigned a value (none $=0$, low $=1$, medium $=2$, high $=3$ ) for both the probability and the intensity of exposure to the chemical(s) of interest. Probability represented the likelihood that the substance was used by a worker in that occupation or industry. Intensity was a combination of the estimated level of exposure and the frequency of use. Algorithms were used to calculate final scores for probability and intensity for each occupation and industry combination. The final probability and intensity scores for occupations in which industry was irrelevant with respect to the agent of interest was the probability value for that occupation squared. For occupations in which the industry was relevant, the final probability score was calculated by multiplying the probability value of the occupation by the probability value for the industry in which the job was held. The final scores for probability and intensity were divided into the 4 levels of none, low, medium, and high ( $0,1-2,3-4,6-9$, respectively) (20). For the jobs held by the subjects, examples of those involving a high probability of exposure to PAH are bus, truck and stationary engine mechanics, molding and casting machine operators, and garage- and service-stationrelated occupations (these occupations also involved exposure to benzene). Participants who had exposure to PAH, but not to benzene held jobs such as traffic, 
shipping and receiving clerks, and inspectors, testers and graders in the motor vehicles industry (these jobs have a low probability and intensity of exposure). Jobs involving exposure to benzene, but not to $\mathrm{PAH}$, which were held by the subjects included clinical laboratory technologists and technicians, painters, sculptors, craft-artists and artist print makers, and assemblers in the motor vehicles industry (these jobs had a medium probability).

\section{Analysis}

Unconditional logistic regression was used to estimate crude and adjusted odds ratios (OR) and $95 \%$ confidence intervals (95\% CI) (21). The adjusted model for each analysis included the exposure variable and age at first live birth (4 dummy variables: $14-21,22-24,25-36$, never), history of benign breast disease (no, yes), months of lactation $(<0.5, \geq 0.5)$, and history of breast cancer in a first-degree relative (no, yes) as categorical variables, plus age, number of years of education, Quetelet index, and age at menarche as continuous variables. Risks were first estimated in relation to each exposure regardless of the other, each exposure independent of the other, and both exposures together. However, the small number of subjects exposed exclusively to PAH did not permit more detailed analyses for this group.

Analyses were carried out according to duration, average probability, average intensity of exposure, and cumulative exposure, with those never exposed to PAH or benzene in the reference group. Duration of exposure to each substance was calculated by summing the years employed in each full-time and $1 / 2$ the years employed in each part-time job involving the exposure. The average probability of each exposure was calculated by summing the probability scores weighted by the duration of employment in each job for full-time jobs and $1 / 2$ the duration for part-time jobs then divided by total duration for that exposure. The intensity was also an average weighted by duration. For both probability and intensity, the medium- and high-level categories were collapsed because of the small numbers of participants with these assigned levels. Cumulative exposure to each chemical of interest was the product of the intensity score and the duration in years for full-time jobs or $1 / 2$ the duration in years for part-time jobs, summed over all jobs in a participant's occupational history. Cut points for cumulative exposure scores were based on the distribution of the scores of the referents. The women for whom the total duration of employment in jobs was unknown were excluded from these analyses.

Latency was defined as the number of years between the first year of employment in an exposed occupation and the reference date. The reference date for cases was the date of diagnosis, and for referents it was the date their interview was completed.

\section{Results}

Only adjusted odds ratios have been reported in this article since the crude and adjusted risk estimates were similar. Premenopausal breast cancer was associated with "ever" being exposed among all the exposure groups except the group of women exposed exclusively to $\mathrm{PAH}$ (table 1). Other than this group, the relative risks were similar among the groups. When we stratified cases by ER status, the risk estimate for exposure exclusively to PAH was higher for ER-negative than for ER-positive tumors. We found a greater risk for ER-positive tumors for the other groups that included women with PAH exposure.

The risk of premenopausal breast cancer by gradient of exposure to PAH is shown in table 2. Duration of exposure was not related to risk. Risk increased with probability and intensity of exposure to PAH. However, the confidence intervals included unity. The risk did not increase in relation to the cumulative exposure to PAH. The subsets of cases with $10-19$ or $\geq 20$ years of latency did not have a greater risk than all the cases, including those more recently exposed.

Table 1. Risk of premenopausal breast cancer associated with having ever been occupationally exposed to polycyclic aromatic hydrocarbons (PAH) and benzene. Odds ratios (OR) adjusted for age, years of education, age at first birth, age at menarche, history of benign breast disease, history of breast cancer in a first-degree relative, Quetelet index, and months of lactation. ( $E R=$ estrogen receptor, $95 \%$ $\mathrm{Cl}=95 \%$ confidence interval)

\begin{tabular}{|c|c|c|c|c|c|c|c|c|c|c|}
\hline \multirow{2}{*}{$\begin{array}{l}\text { Occupational exposure } \\
\text { to PAH and benzene }\end{array}$} & \multirow[t]{2}{*}{ Referents (N) } & \multicolumn{3}{|c|}{ Total cases ${ }^{a}$} & \multicolumn{3}{|c|}{ ER-positive cases } & \multicolumn{3}{|c|}{ ER-negative cases } \\
\hline & & $\begin{array}{c}\text { Cases } \\
(\mathrm{N})\end{array}$ & $\mathrm{OR}$ & $95 \% \mathrm{Cl}$ & $\begin{array}{c}\text { Cases } \\
(\mathbf{N})\end{array}$ & $\mathrm{OR}$ & $95 \% \mathrm{Cl}$ & $\begin{array}{c}\text { Cases } \\
(\mathrm{N})\end{array}$ & $\mathrm{OR}$ & $95 \% \mathrm{Cl}$ \\
\hline Never & 270 & 236 & 1.00 & - & 102 & 1.00 & - & 66 & 1.00 & - \\
\hline $\mathrm{PAH}$ & 25 & 38 & 1.82 & $1.02-3.16$ & 20 & 2.27 & $1.14-4.54$ & 8 & 1.12 & $0.47-2.64$ \\
\hline Benzene & 35 & 56 & 1.91 & $1.18-3.08$ & 28 & 2.29 & $1.27-4.13$ & 12 & 1.49 & $0.74-3.02$ \\
\hline Exclusively PAH & 6 & 6 & 1.01 & $0.55-3.45$ & 2 & 0.78 & $0.12-5.03$ & 3 & 1.97 & $0.46-8.52$ \\
\hline PAH and benzene & 19 & 32 & 2.01 & $1.08-3.75$ & 18 & 2.54 & $1.28-5.04$ & 7 & 1.28 & $0.52-3.15$ \\
\hline Exclusively benzene & 16 & 24 & 1.70 & $1.17-2.92$ & 10 & 1.74 & $0.72-4.21$ & 7 & 2.20 & $0.87-5.53$ \\
\hline
\end{tabular}

a This includes 68 cases with missing ER status. 
Table 2. Premenopausal breast cancer risk by gradient of exposure to polycyclic aromatic hydrocarbons (PAH). Odds ratios (OR) adjusted for age, years of education, age at first birth, age at menarche, history of benign breast disease, history of breast cancer in a first-degree relative, Quetelet index, and months of lactation. ( $E R=$ estrogen receptor, $95 \% \mathrm{Cl}=95 \%$ confidence interval)

\begin{tabular}{|c|c|c|c|c|c|c|c|c|c|c|}
\hline \multirow{2}{*}{$\begin{array}{l}\text { Occupational } \\
\text { exposure to PAH }\end{array}$} & \multirow[t]{2}{*}{ Referents (N) } & \multicolumn{3}{|c|}{ Total cases ${ }^{a}$} & \multicolumn{3}{|c|}{ ER-positive cases } & \multicolumn{3}{|c|}{ ER-negative cases } \\
\hline & & $N$ & OR & $95 \% \mathrm{Cl}$ & $\mathrm{N}$ & $\mathrm{OR}$ & $95 \% \mathrm{Cl}$ & N & $\mathrm{OR}$ & $95 \% \mathrm{Cl}$ \\
\hline Unexposed & 270 & 236 & 1.00 & - & 102 & 1.00 & - & 66 & 1.00 & - \\
\hline \multicolumn{11}{|l|}{ Duration ${ }^{b}$} \\
\hline$<4$ years & 10 & 19 & 2.25 & $0.99-5.09$ & 11 & 2.98 & $1.14-1.75$ & 4 & 1.53 & $0.45-5.19$ \\
\hline$\geq 4$ years & 14 & 18 & 1.49 & $0.70-3.18$ & 9 & 1.89 & $0.74-4.81$ & 4 & 0.97 & $0.28-3.28$ \\
\hline \multicolumn{11}{|l|}{ Average probability ${ }^{\circ}$} \\
\hline Low & 16 & 23 & 1.56 & $0.78-3.12$ & 12 & 2.00 & $0.85-4.68$ & 7 & 1.33 & $0.30-5.85$ \\
\hline Medium-to-high & 8 & 14 & 2.40 & $0.96-6.01$ & 8 & 3.21 & $1.12-9.19$ & 1 & 0.99 & $0.22-4.34$ \\
\hline \multicolumn{11}{|l|}{ Intensity ${ }^{b}$} \\
\hline Low & 17 & 26 & 1.65 & $0.85-3.21$ & 15 & 2.39 & $1.07-5.33$ & 7 & 1.54 & $0.58-4.10$ \\
\hline Medium-to-high & 7 & 11 & 2.25 & $0.82-6.13$ & 5 & 2.37 & $0.61-5.29$ & 1 & 0.50 & $0.06-4.31$ \\
\hline \multicolumn{11}{|c|}{ Cumulative exposureb } \\
\hline Low & 13 & 26 & 2.10 & $1.07-4.53$ & 14 & 1.07 & $1.23-6.88$ & 7 & 1.77 & $0.65-4.83$ \\
\hline Medium-to-high & 11 & 11 & 1.30 & $0.54-3.17$ & 6 & 1.67 & $0.54-4.93$ & 1 & 0.38 & $0.05-3.10$ \\
\hline \multicolumn{11}{|l|}{ Latency } \\
\hline $10-19$ years & 10 & 12 & 1.48 & $0.59-3.71$ & 7 & 1.98 & $0.67-5.98$ & 2 & 0.63 & $0.12-3.24$ \\
\hline$\geq 20$ years & 8 & 13 & 1.78 & $0.70-4.52$ & 7 & 2.22 & $0.73-6.75$ & 4 & 2.13 & $0.59-7.67$ \\
\hline
\end{tabular}

a This includes 68 cases with missing ER status.

${ }^{b}$ One case and one referent with missing information on duration of employment in exposed jobs were excluded.

Due to small numbers, and the high correlation between PAH and benzene for some exposure gradients, we gained little insight into the independent role $\mathrm{PAH}$ played in the risk of breast cancer after stratifying by gradient of exposure to benzene. Adjustment of the analyses for level of benzene exposure produced unstable results, but the risk estimates did not change for probability (low: OR $1.47,95 \%$ CI $0.62-3.50$; high: OR 2.37 , 95\% Cr $0.94-5.94$ ) or duration ( $<4$ years: OR $2.78,95 \%$ CI $0.39-19.76 ; \geq 4$ years: OR $1.71,95 \%$ CI $0.70-4.23$ ). The odds ratios related to intensity decreased (low: OR $0.98,95 \%$ CI 0.25 - 3.76; high: OR $1.63,95 \%$ CI $0.53-$ 5.04) (data not shown). The analyses of $\mathrm{PAH}$ probability of exposure by intensity of exposure showed that the highest risk was associated with having both a mediumto-high probability and intensity level of exposure (OR 2.63,95\% CI 0.92-7.53) (data not shown).

When the analyses were stratified by tumor ER status (table 2), we found a statistically significant 3-fold increase in the risk of ER-positive tumors in association with medium-to-high probability of exposure to $\mathrm{PAH}$. The risk increased in relation to probability and cumulative exposure, but an inverse relationship was observed for duration. There were no significant elevations in the risk of ER-negative tumors observed in relation to $\mathrm{PAH}$ exposure, but the sample sizes were very small.

Table 3 presents the risk of premenopausal breast cancer according to exposure to benzene. One-half of the benzene-exposed women were also exposed to PAH. The risk increased with both the duration and probability of exposure. A 2-fold increase in risk was observed for both medium-to-high probability and longer duration of exposure. The risk was elevated for low, but not for medi- um-to-high, intensity. Adjustment for the level of PAH exposure had little effect on the results (duration: $<4$ years OR $1.42,95 \%$ CI $0.26-7.64 ; \geq 4$ years OR 1.71 , 95\% CI $0.70-4.23)$; intensity: low OR $3.48,95 \% \mathrm{CI}$ $0.93-5.48$; high OR $1.63,95 \%$ CI $0.53-5.04$ ) (data not shown).

The risk of ER-positive breast tumors was higher than for ER-negative tumors for duration and probability of exposure to benzene. We observed a 3 -fold significant increase in the risk of ER-positive tumors for a low intensity of exposure to benzene. High intensity of exposure was not related to the risk of ER-positive tumors. There were no significant increases in the risk related to ER-negative tumors.

When women who had PAH exposure were excluded (table 4), exposure to benzene for at least 4 years was associated with a 3 -fold increase in risk. The risk increased with cumulative exposure to benzene in this group. The confidence intervals, however, included unity. The risk related to benzene exposure increased for a latency period of $\geq 20$ years when the analyses were restricted to women who were never exposed to PAH.

There were no apparent differences in risk between ER-positive and ER-negative breast tumors in relation to benzene exposure when the women exposed to PAH were excluded.

\section{Discussion}

Using job exposure matrices (19) and lifetime occupational histories, we found an association between premenopausal breast cancer risk and occupational exposure to 
benzene. Analyses examining risk related to $\mathrm{PAH}$ independent of benzene were not possible because of the small sample size of those exposed exclusively to PAH. A comparison of risk among several exposure groups showed that women exposed to both PAH and benzene had the highest risk. However, nearly all these women had a medium-to-high probability of exposure to benzene, and this exposure may explain the higher risk seen in this group. There was some evidence of a dose-response relationship between risk of premenopausal breast cancer and benzene exposure according to the duration and probability analyses. There was also an indication that a latency period of $\geq 20$ years may exist for benzenerelated breast cancer. Analyses of the probability and intensity of PAH exposure seemed to suggest a possible dose-response relationship between exposure to $\mathrm{PAH}$ and risk. Adjusting for the level of exposure to benzene in most analyses of risk related to the gradient of exposure to PAH did not change the results. Similarly, adjustment for level of exposure to PAH did not change the results of analyses for risk by gradient of exposure to benzene. Although based on small numbers, these findings

Table 3. Premenopausal breast cancer risk by gradient of exposure to benzene. Odds ratios $(O R)$ adjusted for age, years of education, age at first birth, age at menarche, history of benign breast disease, history of breast cancer in a first-degree relative, Quetelet index, and months of lactation. ( $\mathrm{ER}=$ estrogen receptor, $95 \% \mathrm{Cl}=95 \%$ confidence interval)

\begin{tabular}{|c|c|c|c|c|c|c|c|c|c|c|}
\hline \multirow{2}{*}{$\begin{array}{l}\text { Occupational } \\
\text { exposure to } \\
\text { benzene }\end{array}$} & \multirow[t]{2}{*}{ Referents (N) } & \multicolumn{3}{|c|}{ Total cases $^{a}$} & \multicolumn{3}{|c|}{ ER-positive cases } & \multicolumn{3}{|c|}{ ER-negative cases } \\
\hline & & $N$ & $\mathrm{OR}$ & $95 \% \mathrm{Cl}$ & $N$ & OR & $95 \% \mathrm{Cl}$ & $N$ & $\mathrm{OR}$ & $95 \% \mathrm{Cl}$ \\
\hline Unexposed & 270 & 236 & 1.00 & - & 102 & 1.00 & - & 66 & 1.00 & - \\
\hline \multicolumn{11}{|l|}{ Duration } \\
\hline$<4$ years & 20 & 25 & 1.47 & $0.78-2.79$ & 13 & 1.80 & $0.82-3.95$ & 5 & 0.98 & $0.33-2.86$ \\
\hline$\geq 4$ years & 15 & 30 & 2.57 & $1.23-4.73$ & 15 & 2.97 & $1.32-6.69$ & 7 & 1.93 & $0.71-5.28$ \\
\hline \multicolumn{11}{|l|}{ Average probability } \\
\hline Low & 8 & 12 & 1.64 & $0.64-4.21$ & 9 & 2.91 & $1.04-8.23$ & 1 & 0.41 & $0.09-1.82$ \\
\hline Medium-to-high & 27 & 43 & 1.95 & $1.14-3.33$ & 19 & 2.09 & $1.07-4.08$ & 11 & 1.73 & $0.40-7.61$ \\
\hline \multicolumn{11}{|l|}{ Average Intensity ${ }^{b}$} \\
\hline Low & 19 & 36 & 2.36 & $1.30-4.30$ & 22 & 3.25 & $1.62-6.53$ & 7 & 1.40 & $0.53-3.67$ \\
\hline Medium-to-high & 16 & 19 & 1.25 & $0.60-2.64$ & 6 & 1.08 & $0.38-3.05$ & 5 & 1.38 & $0.46-4.12$ \\
\hline \multicolumn{11}{|l|}{ Cumulative exposureb } \\
\hline Low & 17 & 29 & 1.81 & $0.95-3.47$ & 14 & 2.03 & $0.92-4.50$ & 7 & 1.45 & $0.54-3.87$ \\
\hline Medium-to-high & 18 & 26 & 1.93 & $1.00-3.72$ & 14 & 2.59 & $1.16-5.79$ & 5 & 1.29 & $0.44-3.79$ \\
\hline \multicolumn{11}{|l|}{ Latency } \\
\hline $10-19$ years & 12 & 17 & 1.86 & $0.82-4.19$ & 7 & 1.71 & $0.60-4.89$ & 6 & 2.30 & $0.76-7.02$ \\
\hline$\geq 20$ years & 18 & 25 & 1.52 & $0.79-2.93$ & 13 & 1.89 & $0.86-4.14$ & 3 & 0.61 & $0.17-2.20$ \\
\hline
\end{tabular}

a This includes 68 cases with missing ER status.

${ }^{b}$ One case with missing information on duration of employment in exposed jobs was excluded.

Table 4. Premenopausal breast cancer risk among women who were never exposed to polycyclic aromatic hydocarbons (PAH) by gradient of exposure to benzene. Odds ratios (OR) adjusted for age, years of education, age at first birth, age at menarche, history of benign breast disease, history of breast cancer in a first-degree relative,, Quetelet index, and months of lactation. (ER $=$ estrogen receptor, $95 \% \mathrm{Cl}=95 \%$ confidence interval)

\begin{tabular}{|c|c|c|c|c|c|c|c|c|c|c|}
\hline \multirow{2}{*}{$\begin{array}{l}\text { Occupational } \\
\text { exposure to } \\
\text { benzene }\end{array}$} & \multirow[t]{2}{*}{ Referents (N) } & \multicolumn{3}{|c|}{ Total cases ${ }^{\mathrm{a}}$} & \multicolumn{3}{|c|}{ ER-positive cases } & \multicolumn{3}{|c|}{ ER-negative cases } \\
\hline & & $N$ & OR & $95 \% \mathrm{Gl}$ & $N$ & $\mathrm{OR}$ & $95 \% \mathrm{Cl}$ & N & $\mathrm{OR}$ & $95 \% \mathrm{Cl}$ \\
\hline Unexposed & 270 & 236 & 1.00 & - & 102 & 1.00 & - & 66 & 1.00 & - \\
\hline \multicolumn{11}{|l|}{ Duration } \\
\hline$<4$ years & 10 & 8 & 0.80 & $0.30-2.16$ & 5 & 0.94 & $0.30-2.94$ & 2 & 0.62 & $0.12-3.16$ \\
\hline$\geq 4$ years & 6 & 16 & 3.38 & $1.25-9.17$ & 5 & 4.65 & $1.08-19.99$ & 5 & 6.36 & $1.47-27.4$ \\
\hline \multicolumn{11}{|l|}{ Probability } \\
\hline Low & 7 & 8 & 1.22 & $0.42-3.56$ & 5 & 1.71 & $0.50-1.71$ & 1 & 0.46 & $0.05-4.45$ \\
\hline Medium-to-high & 9 & 16 & 2.14 & $0.89-5.12$ & 5 & 1.78 & $0.56-5.70$ & 6 & 3.40 & $1.01-10.81$ \\
\hline \multicolumn{11}{|l|}{ Intensity } \\
\hline Low & 8 & 16 & 2.38 & $0.97-5.87$ & 8 & 2.75 & $0.95-7.96$ & 3 & 1.64 & $0.80-7.11$ \\
\hline Medium-to-high & 8 & 8 & 1.07 & $0.37-3.07$ & 2 & 0.63 & $0.11-3.45$ & 4 & 2.21 & $0.60-8.15$ \\
\hline \multicolumn{11}{|l|}{ Cumulative } \\
\hline Low & 10 & 13 & 1.43 & $0.59-3.47$ & 5 & 1.44 & $0.45-4.58$ & 3 & 1.32 & $0.32-5.46$ \\
\hline Medium-to-high & 6 & 11 & 2.21 & $0.77-6.36$ & 5 & 2.27 & $0.60-8.65$ & 4 & 2.77 & $0.71-10.87$ \\
\hline \multicolumn{11}{|l|}{ Latency } \\
\hline $10-19$ years & 6 & 5 & 1.23 & $0.34-4.46$ & 1 & 0.66 & $0.07-6.18$ & 3 & 3.33 & $0.72-15.28$ \\
\hline$\geq 20$ years & 8 & 16 & 2.09 & $0.85-5.14$ & 7 & 1.98 & $0.66-5.94$ & 3 & 1.39 & $0.33-5.78$ \\
\hline
\end{tabular}

a This includes 68 cases with missing estrogen receptor status. 
suggest a possible independent relationship between risk and benzene exposure, and some evidence for an association between risk and PAH exposure, although there was no elevation of risk among the women exposed to $\mathrm{PAH}$ but not benzene.

When the analyses were stratified by tumor ER status, PAH exposure appeared to be more strongly related to the risk of ER-positive tumors than to the risk of all breast tumors or to the risk of ER-negative tumors. There was no striking difference in risk by ER status when PAH-exposed women were excluded from the benzeneexposed group.

Among the small group exposed exclusively to PAH, a greater risk for ER-negative than ER-positive tumors was found. Our results must be interpreted with caution as they are based on small sample sizes, especially the analyses stratified by ER status. The results of the analyses for risk related to $\mathrm{PAH}$ exposure should especially be interpreted with caution, as we could not examine risk independently of benzene exposure. The evidence from this study of an association between risk and occupational exposure to PAH is therefore limited.

The suggested increased risk associated with benzene and possibly PAH exposure seen in our study is consistent with animal data which demonstrated that benzene, $\mathrm{BaP}, \mathrm{DMBA}$ and DB[ah]A are mammary carcinogens. Because of the high proliferative activity of epithelial cells of the mammary gland and susceptibility to chemical carcinogens, mammary terminal duct lobular units (TDLU) are likely target tissues for tumorigenesis (22). Experimental investigations using human breast tissue have demonstrated the specificity of $\mathrm{BaP}$ for mammary TDLU (22). Both BaP and benzene have been detected in human breast milk $(23,24)$. The results of our analyses stratified by ER status were consistent with the findings of experimental studies by Telang et al $(8,9)$, who found that both BaP and DMBA altered the metabolism of estradiol and led to an increased formation of the estradiol metabolite $16 \alpha$-hydroxyestrone, which bonds strongly to the estrogen receptor and has been shown to promote cancer (9).

Cigarette smoke is a source of $\mathrm{PAH}$ and benzene exposure, but it has not been found to increase premenopausal breast cancer risk in these data (25) or in other studies (26). However, other sources of PAH and benzene may still be risk factors for breast cancer. The frequency of PAH-DNA adducts has been found to be associated with breast cancer risk (6) and occupational exposure to PAH (3).

Breast cancer was not elevated among the women with potential occupational exposure to $\mathrm{PAH}$ in 2 previous epidemiologic studies $(13,14)$. Previous epidemiologic studies of benzene exposure and breast cancer are inconsistent. Mikoczy et al (15) found that workers exposed to benzene and other potential carcinogens in leath- er tanneries had a higher than expected incidence of breast cancer. The results of the present study are not consistent with those of 2 cohort studies. Paci et al (16) found that occupational exposure to benzene did not increase breast cancer mortality in a shoe manufacturing plant, and Yin et al (17) found no association in a large cohort study of workers exposed to benzene.

When our results are interpreted, several issues need to be considered. The response rates for both the cases and referents were low. Among the cases, failure to participate was primarily related to physician nonresponse $(12 \%)$ or refusal to allow us to contact patients $(13 \%)$ (18). It may be that those refusals were related to disease stage, physicians refusing to allow us to contact patients with more advanced disease. If so, our results would not generalize to that group. Although it is unlikely that participation among referents was related to specific occupational exposures, it is possible that participation was related to socioeconomic status, of which occupation is a determining factor. If women of higher socioeconomic status in professional jobs had been more likely to participate as referents than women of lower socioeconomic status in blue-collar jobs, the risks observed in our study may have been overestimated. If professional women had been less likely to participate, our odds ratios may have been underestimated. Although we had no occupational data on nonrespondents, the results of a study of a subset of nonrespondents and participants showed that there were no differences with regard to dietary and smoking habits between the women who participated in the study and those who did not (18). Hence the participants did not appear to be more health conscious than the nonrespondents.

Since we had no direct measures of exposure, some undetermined degree of misclassification existed in this study. To date, the comparability between exposure classification according to the matrices used and the expert assessment of exposure on a case-by-case basis has not been done. Furthermore, the risks shown to be associated with benzene could be related to other solvent exposures correlated with benzene exposure, as other solvents have been associated with breast cancer (27).

Finally, we did not have the power to detect small elevations in risk due to the small numbers of exposed women. This was more of a problem in the more refined analyses of duration, probability, and intensity and in the analyses stratified by ER status.

Although our study possessed some significant limitations, it had important strengths. The literature on occupational risk factors for female breast cancer is sparse and even fewer studies address exposures. The use of a job-exposure matrix allowed us to examine risk by exposures suspected to increase breast cancer risk. The matrix also had features such as probability and intensity scores which refined the exposure assessment. The use 
of gradients of exposure has been shown to improve the accuracy of risk estimates $(28,29)$. Information from lifetime occupational histories enabled us to assess risk for all the participants who had ever been potentially exposed to the agent of interest through an occupation and to analyze the relationship between risk and exposure by various dimensions of exposure with better accuracy than if we had information on only one job. Having a complete occupational history should have eliminated some sources of misclassification because potential exposures were not missed by the omission of some jobs. Additional information from the interviews enabled us to adjust odds ratios for established breast cancer risk factors.

Our results suggest that future research examining breast cancer risk in relation to exposure to benzene is warranted and that distinguishing between ER-positive and ER-negative breast tumors may be important when the risk of breast cancer is examined in association with exposure to PAH. Occupational studies with larger sample sizes and more refined measures of exposure are needed to confirm our findings.

\section{References}

1. Morris JJ, Seifter E. The role of aromatic hydrocarbons in the genesis of breast cancer. Med Hypotheses 1992;38:177-84.

2. Labrèche FP, Goldberg MS. Exposure to organic solvents and breast cancer in women: a hypothesis. Am J Ind Med 1997;32:1-14.

3. Brandt-Rauf PW, Smith S, Perera FP, Nimen HL, Yohannan $\mathrm{W}$, Hemminki $\mathrm{K}$, et al. Serum oncogene proteins in foundry workers. J Soc Occup Med 1990;40(s1):11—4.

4. Perera FP, Estabrook A, Hewer A, Channing K, Runde A, Mooney LA, et al. Carcinogen-DNA adducts in human breast tissue. Cancer Epidemiol Biomarkers Prev 1995;4(s3):2338.

5. Perera FP, Hemminki K, Gryzbowska E. Molecular and genetic damage in humans from environmental pollution in Poland. Nature 1992;360:256-8.

6. Tompa A, Major J, Jakob MG. Monitoring of benzene-exposed workers for genotoxic effects of benzene. Mutat Res 1994;304:159-64.

7. Karacic V, Skenda L, Bosner-Cucancic B, Bogadi-Sare A. Possible genotoxicity in low level benzene exposure. Am J Ind Med 1995;24:379_88.

8. Telang NT, Axelrod DM, Bradlow HL, Osborne MP. Metabolic biotransformation of estradiol in human mammary explant cultures. Ann N Y Acad Sci 1990;586:70-8.

9. Telang NJ, Suto A, Wong GY, Osborne MP, Bradlow HL. Induction by estrogen metabolite 16alpha-hydroxyestrone of genotoxic damage and aberrant proliferation in mouse mammary epithelial cells. JNCI 1992;84:634—8.

10. Davis DL, Bradlow HL, Wolff M, Woodruff T, Hoel DG, Anton-Culver $\mathrm{H}$, et al. Medical hypothesis: xenoestrogens as preventable causes of breast cancer. Environ Health Perspect 1993;101(5):372-377.

11. Dewailly E, Dodin S, Verreault R, Ayotte P, Sauve L, Morin $\mathrm{J}$, et al. High organochlorine body burden in women with estrogen receptor-positive breast cancer. JNCI 1994;86:232 -
4.

12. Mussalo-Rauhamaa H, Pantzar P. Selenium and DDE in breast fat of breast cancer patients: their relationship to hormone receptors in breast tissues. JNCI 1993;85:1964-5.

13. Cantor KP, Stewart PA, Brinton LA, Dosemeci M. Occupational exposures and female breast cancer mortality in the United States. J Occup Environ Med 1995;37:336-48.

14. Solionova LG, Smulevich VB. Mortality and cancer incidence in a cohort of rubber workers in Moscow. Scand J Work Environ Health 1993;19:96-101.

15. Mikoczy Z, Schutz A, Hagmar L. Cancer incidence and mortality among Swedish leather tanners. Occup Environ Health 1994;51:530-5.

16. Paci E, Buiatti E, Costantini AS. Aplastic anemia, leukemia and other cancer mortality in a cohort of shoe workers exposed to benzene. Scand J Work Environ Health 1989;15:313-18.

17. Yin S-N, Hayes RB, Linet MS, Li Gi-L, Dosemeci M, Travis LB, et al. A cohort study of cancer among benzene-exposed workers in China: overall results. Am J Ind Med 1996;29:227-35.

18. Freudenheim JL, Marshall JR, Vena JE, Laughlin R, Brasure $\mathrm{J}$, Swanson MK, et al. Premenopausal breast cancer risk and intake of vegetables, fruits and related nutrients. JNCl 1996;88:340-8.

19. United States Department of Commerce. 1980 census of the population, alphabetical index of industries and occupations. Washington (DC): United States Department of Commerce, 1982. Publication number 80-R3.

20. Gomez MR, Cocco P, Dosemeci M, Stewart PA. Occupational exposure to aliphatic hydrocarbons: job exposure matrix. Am J Ind Med 1994;26:171—83.

21. Breslow NE, Day NE. Statistical methods in cancer research; vol 1 (The analysis of case-control studies). Lyon: International Agency for Cancer Research (IARC), 1980:5-338. IARC scientific publications, no 32.

22. Telang NT, Basu A, Modak MJ. Cellular ras protooncogene expression in human mammary explant cultures. Ann NY Acad Sci 1990;230-7.

23. Madhavan ND, Naidu KA. Polycyclic aromatic hydrocarbons in placenta, maternal blood, umbilical cord blood and milk of Indian women. Hum Exp Toxicol 1995;14:503-6.

24. Pellizzari ED, Hartwell TD, Harris BS, Waddel RD, Whitaker DA, Erikson MD. Purgeable organic compounds in mother's milk. Bull Environ Contam Toxicol 1982;28:322-8.

25. Ambrosone CB, Freudenheim JL, Graham S, Marshall JR, Vena JE; Brasure J, et al. Cigarette smoking, N-Acetyltransferase 2 genetic polymorphisms, and breast cancer risk. JAMA 1996;276:1494-1501.

26. Palmer JR, Rosenberg L. Cigarette smoking and the risk of breast cancer. Epidemiol Rev 1993;15:145-56.

27. Blair A, Hartge P, Stewart PA, McAdams M, Lubin J. Mortality and cancer incidence of aircraft maintenance workers exposed to trichlorethylene and other organic solvents and chemicals: extended follow up. Occup Environ Med 1998;55:161— 71.

28. Blair A, Stewart PA. Do quantitative measurements improve risk estimates in occupational studies of cancer? Am J Ind Med 1992;21:53-63.

29. Dosemeci M, Cocco P, Gòmez M, Stewart PA, Heineman EF. Effects of three features of a job-exposure matrix on risk estimates. Epidemiology 1994;5:124—7.

Received for publication: 30 March 1998 